\title{
Nonmagnetic impurities in two- and three-dimensional Heisenberg antiferromagnets
}

\author{
Tai-Kai Ng \\ Department of Physics, Hong Kong University of Science and Technology, Clear Water Bay Road, Kowloon, Hong Kong
}

(Received 30 July 1996)

\begin{abstract}
In this paper we study in a large- $S$ expansion the effects of substituting spins by nonmagnetic impurities in two- and three-dimensional Heisenberg antiferromagnets in a weak magnetic field. In particular, we demonstrate a mechanism where magnetic moments are induced around nonmagnetic impurities when magnetic field is present. As a result, Curie-type behavior in magnetic susceptibility can be observed well below the Néel temperature, in agreement with what is observed in $\mathrm{La}_{2} \mathrm{Cu}_{1-x} \mathrm{Zn}_{x} \mathrm{O}_{4}$ and $\operatorname{Sr}\left(\mathrm{Cu}_{1-x} \mathrm{Zn}_{x}\right)_{2} \mathrm{O}_{3}$ compounds. [S0163-1829(96)04542-0]
\end{abstract}

Recently, there has been increasing interests in the effects of substituting $\mathrm{Cu}$ by nonmagnetic ions $(\mathrm{Zn})$ in high- $T_{c} \mathrm{cu}-$ prates, where interesting effects were discovered for compounds in the underdoped regime. ${ }^{1}$ In particular, magneticsusceptibility measurements, ${ }^{2}$ and other experiments ${ }^{3}$ seem to indicate that local magnetic moments are generated as $\mathrm{Cu}$ ions were replaced by $\mathrm{Zn}$, both in the underdoped high- $T_{c}$ yttrium and bismuth compounds, ${ }^{2,3}$ and for an undoped lanthanum compound. ${ }^{4}$ Based on resonant-valence band theories of the $t-J$ model, Nagaosa and $\mathrm{Ng}^{5}$ have constructed explanations on how local magnetic moments can be generated in these compounds in the disordered phase of twodimensional antiferromagnets. Their theory is believed to be applicable to the underdoped regime of high- $T_{c}$ compounds. However the theory is not applicable to the La compound where Curie-type behavior is observed in magnetic susceptibility at temperatures well below the Néel temperature where long-range antiferromagnetic order exists. More recently, similar experiment on the two-leg ladder compound $\operatorname{Sr}\left(\mathrm{Cu}_{1-x} \mathrm{Zn}_{x}\right)_{2} \mathrm{O}_{3}$ also indicates formation of local magnetic moments in the spin-gap phase as $\mathrm{Cu}$ ions are replaced by $\mathrm{Zn}$ ions. ${ }^{6}$ The compound was found to order antiferromagnetically at low temperature at $x \sim 0.07 .^{7}$ Surprisingly, Curie behavior in magnetic susceptibility remains even below the Néel-ordering temperature, ${ }^{7}$ indicating that formation of magnetic moments around nonmagnetic impurities is a general phenomenon in Néel state of quantum antiferromagnets.

In the presence of long-range antiferromagnetic order, the low-temperature properties of clean antiferromagnets can be understood at least qualitatively using a semiclassical theory ( $1 / S$ expansion). In this paper we shall generalize this approach to include the effects of nonmagnetic impurities in the presence of a uniform magnetic field. Notice that in the absence of magnetic field, a similar study has been performed by Bulut et al., ${ }^{8}$ where no "free", magnetic moments were found to be induced around nonmagnetic impurities. We shall show that local magnetic moments can be induced by nonmagnetic impurities once magnetic field is applied on the system through a novel mechanism. Notice that strictly speaking, thermal fluctuations destroy long-range magnetic order at two dimensions at any finite temperature. Thus our analysis at two dimensions (2D) can only be applied to layered systems like lanthanum cuprates where the magnetic coupling between different layers is much weaker than intralayer coupling.
To begin with, we first consider the zero temperature problem of classical spins interacting antiferromagnetically under a uniform magnetic field $B$, with a single nonmagnetic impurity replacing spin at site $i_{0}$. We shall consider the Néel state in the absence of magnetic field to be ordered in the $z$ direction, with the uniform magnetic field $B$ applied in the $+x$ direction. The classical ground state in the absence of magnetic field has spins all pointing in $+z$ direction for spins in the $A$ sublattice and spins all pointing in the $-z$ direction for spins in the $B$ sublattice. In the presence of a magnetic field, the spins tilt to the $+x$ direction to minimize the magnetic energy. Let $\theta_{i}^{A}$ be the angle tilted away from the $z$ axis for spin on site $i$ on the $A$ sublattice and $\theta_{j}^{B}$ be the corresponding angle for spin on site $j$ on the $B$ sublattice. It is easy to show that the classical energy $E_{\mathrm{cl}}$ is given in the limit when $\theta^{A(B)}$ are small (weak $B$-field limit) by

$$
\begin{aligned}
E_{\mathrm{cl}} / S^{2}= & -\sum_{\left\langle i \neq i_{0}, j\right\rangle}\left(1-\frac{\left(\theta_{i}^{A}+\theta_{j}^{B}\right)^{2}}{2}\right)-B^{\prime} \sum_{i \neq i_{0}} \theta_{i}^{A} \\
& -B^{\prime} \sum_{j} \theta_{j}^{B},
\end{aligned}
$$

where $\left\langle i \neq i_{0}, j\right\rangle$ are nearest-neighbor sites in the square (cubic) lattice excluding contributions from the nonmagnetic impurity at site $i_{0}$ and $B^{\prime}=g \mu_{B} B / S$. Notice that the only effect of nonmagnetic impurity is to remove the spin at site $i_{0}$ in our theory. We have also set the spin-coupling $J=1$ in Eq. (1). Notice that the energy expression for $E_{\mathrm{cl}}$ is valid only to order $O\left(B^{2}\right)$.

Next we consider the continuum limit of the energy expression (1). Introducing symmetric and antisymmetric angle variables

$$
\begin{aligned}
\theta_{s}(\vec{x}) & =S\left[\theta^{A}(\vec{x})+\theta^{B}(\vec{x})\right], \\
\theta_{a}(\vec{x}) & =\frac{S}{2}\left[\theta^{A}(\vec{x})-\theta^{B}(\vec{x})\right],
\end{aligned}
$$

we obtain after some straightforward algebra 


$$
\begin{aligned}
E_{\mathrm{cl}}= & \int \frac{d^{d} x}{a_{o}^{d}}\left[(d) \theta_{s}(\vec{x})^{2}-B^{\prime} S \theta_{s}(\vec{x})+\left[\nabla \theta_{a}(\vec{x})\right]^{2}\right. \\
& +\frac{\theta\left(x_{<}\right)}{v_{a}}\left[\theta_{s}(\vec{x}) \hat{a}_{o} \cdot \nabla \theta_{a}(\vec{x})-\theta_{a}(\vec{x}) \hat{a}_{o} \cdot \nabla \theta_{s}(\vec{x})\right]+E_{0},
\end{aligned}
$$

where $E_{0}=-N S^{2} d / 2, N=$ number of sites in the system, $a_{o}$ is lattice spacing and $\hat{a}_{o}$ is a radial vector pointing away from $i_{0} . v_{a}$ is the volume enclosed by the sphere (circle in 2D) with radius $a_{o}, x_{<}=a_{o}-\left|\vec{x}-\vec{x}_{0}\right|$. For an impurity located at the $B$ sublattice, $\hat{a}_{o} \rightarrow-\hat{a}_{o}$.

The various terms appearing in Eq. (3) can be understood rather easily. First of all, in the limit $a_{o} \rightarrow 0, x_{i} \rightarrow x_{j}$ in Eq. (1) and the only contribution to $E_{\mathrm{cl}}$ would be the first two $\theta_{s}$ terms in Eq. (3). It is also clear that in the limit $a_{o} \rightarrow 0$, terms proportional to $\theta_{a}$ or $\theta_{a}^{2}$ do not appear in $E_{\mathrm{cl}}$, and the only contribution from $\theta_{a}$ can appear as $\left(\nabla \theta_{a}\right)^{2}$ only. The appearance of a nonmagnetic impurity at site $i_{0}$ breaks the symmetry between the $A$ and $B$ sublattices and introduces local coupling between the $\theta_{s}$ and $\theta_{a}$ fields. Notice that additional coupling between $\theta_{a}$ and $\theta_{s}$ fields will appear if we take into account higher-order terms in $B$ in our energy expression $E_{\mathrm{cl}}$. However, in the absence of impurities, these terms do not break the symmetry between the $A$ and $B$ sublattices or the symmetry of interchanging $\theta^{A}$ and $\theta^{B}$ fields.

Minimizing $E_{\mathrm{cl}}$ with respect to the $\theta_{s}$ field we obtain

$$
\theta_{S}(\vec{x})=\frac{1}{2 d}\left(B^{\prime} S-\frac{2 \theta\left(x_{<}\right)}{v_{a}} \hat{a}_{o} \cdot \nabla \theta_{a}(\vec{x})\right) .
$$

Putting $\theta_{s}$ back into $E_{\mathrm{cl}}$ and minimizing with respect to $\theta_{a}$ field, we obtain for $\left|\vec{x}-\vec{x}_{0}\right| \geqslant a_{o}$,

$$
\nabla^{2} \theta_{a}(\vec{x})=\frac{2 B^{\prime} S a_{o}}{d v_{a}} \delta^{d}\left(\left|\vec{x}-\vec{x}_{0}\right|-a_{o}\right)
$$

Notice that in the presence of magnetic field, the nonmagnetic impurity acts as a source term for the $\theta_{a}$ field of strength $2 B^{\prime} S$ in the limit $a_{o} \rightarrow 0$. As a result, $\theta_{a}(\vec{x}) \sim\left(2 B^{\prime} S\right) \ln \left(\left|\vec{x}-\vec{x}_{0}\right|\right)$ in two dimensions, and the corresponding "electric-field" energy cost $\sim \int d^{2} x\left(\nabla \theta_{a}\right)^{2}$ diverges logarithmically as the size of system goes to infinity.

The divergence of magnetic energy to order $O\left(B^{2}\right)$ indicates that the (classical) response of a nonmagnetic impurity to external magnetic field is intrinsically nonlinear ${ }^{9}$ and suggests that quantum effect may play an important role in determining the correct response of nonmagnetic impurities to external magnetic field. In the following we shall derive in the continuum limit, the $1 / S$ (spin wave) Lagrangian in the presence of background $\theta_{s}$ and $\theta_{a}$ fields, and shall show that the infrared divergence in classical energy can be cured by formation of local magnetic moment around nonmagnetic impurity once quantum effects are considered.

To derive the spin-wave Hamiltonian in the presence of magnetic field, we rotate our coordinate system locally on each site such that the local $z$ axis is always along the "classical" spin direction. In this coordinate system, the Hamiltonian becomes

$$
\begin{aligned}
H= & \sum_{\left\langle i \neq i_{0}, j\right\rangle}\left[\cos \left(\theta_{i}+\theta_{j}\right)\left(S_{i}^{(z)} S_{j}^{(z)}+S_{i}^{(x)} S_{j}^{(x)}\right)+S_{i}^{(y)} S_{j}^{(y)}\right. \\
& \left.+\sin \left(\theta_{i}+\theta_{j}\right)\left(S_{i}^{(z)} S_{j}^{(x)}-S_{i}^{(x)} S_{j}^{(z)}\right)\right] \\
& -g \mu_{B} B \sum_{i \neq i_{0}}\left(S_{i}^{(z)} \sin \theta_{i}+S_{i}^{(x)} \cos \theta_{i}\right) \\
& -g \mu_{B} B \sum_{j}\left(\cos \theta_{j} S_{j}^{(x)}-\sin \theta_{j} S_{j}^{(z)}\right)
\end{aligned}
$$

The Hamiltonian can be rewritten in the Schwinger-boson representation of spins in the usual way. In the large- $S$ limit, we may write

$$
Z_{i \uparrow}^{A}=\bar{Z}_{i \uparrow}^{A}=Z_{j \downarrow}^{B}=\bar{Z}_{j \downarrow}^{B}=\sqrt{(2 S)},
$$

where $\bar{Z}(Z)_{i \sigma}^{\alpha}$ 's are spin $\sigma$ Schwinger-boson creation (annihilation) operators for site $i$ on sublattice $\alpha$. Expanding the Hamiltonian to order $O(S)$, we obtain to order $O\left(B^{2}\right)$,

$$
H=E_{\mathrm{cl}}+H_{1 / S},
$$

where

$$
\begin{aligned}
H_{1 / S}= & S \sum_{\left\langle i \neq i_{0}, j\right\rangle}\left[\left(1-\frac{\left(\theta_{i}+\theta_{j}\right)^{2}}{2}\right)\left(\bar{Z}_{i \downarrow}^{A} Z_{i \downarrow}^{A}+\bar{Z}_{j \uparrow}^{B} Z_{j \uparrow}^{B}\right)\right. \\
& \left.+\left(1-\frac{\left(\theta_{i}+\theta_{j}\right)^{2}}{4}\right)\left(Z_{i \downarrow}^{A} Z_{j \uparrow}^{B}+\bar{Z}_{i \downarrow}^{A} \bar{Z}_{j \uparrow}^{B}\right)\right] \\
& +g \mu_{B} B \sum_{i \neq i_{0}}\left(\theta_{i}\right) \bar{Z}_{i \downarrow}^{A} Z_{i \downarrow}^{A}+g \mu_{B} B \sum_{j}\left(\theta_{j}\right) \bar{Z}_{j \uparrow}^{B} Z_{j \uparrow}^{B} .
\end{aligned}
$$

To further analyze our system we again go to the continuum limit. Introducing symmetric and antisymmetric boson fields

$$
\begin{aligned}
& \phi(\vec{x})=\frac{1}{\sqrt{2}}\left[Z_{\downarrow}^{A}(\vec{x})-\vec{Z}_{\uparrow}^{B}(\vec{x})\right], \\
& \pi(\vec{x})=\frac{1}{\sqrt{2}}\left[Z_{\downarrow}^{A}(\vec{x})+\vec{Z}_{\uparrow}^{B}(\vec{x})\right],
\end{aligned}
$$

and integrating out the $\pi(\vec{x})$ field, we obtain in the continuum limit an effective Lagrangian for the $\phi(\vec{x})$ field. ${ }^{10,11}$ Details of the technique can be found in Ref. 10 and we shall not repeat them here. For $\vec{x}$ away from the impurity site $\left(a_{o}<\left|\vec{x}-\vec{x}_{0}\right|\right)$, we obtain in imaginary time

$$
\begin{aligned}
L_{1 / S} \sim & \int d \tau \int d^{d} x \frac{1}{4 d S}\left[\left(\frac{\partial}{\partial \tau}-e \theta_{a}\right) \phi^{+}\left(\frac{\partial}{\partial \tau}+e \theta_{a}\right) \phi\right. \\
& \left.+m^{2} \phi^{+} \phi+c^{2}|\nabla \phi|^{2}\right],
\end{aligned}
$$

where $e=B^{\prime}$ and $m^{2}=S^{2} B^{\prime 2} / 2 . c^{2} \sim 4 d(J S)^{2}$ is the spinwave velocity. We have set $\theta_{S}(\vec{x})=B^{\prime} S / 2 d$ and have neglected $\left(\nabla \theta_{a}\right)^{2}$ terms in deriving $L_{1 / S}$. The latter is of higher order $[O(1 / S)]$ compared with the corresponding term in 
$E_{\mathrm{cl}}$. The most striking feature of the effective Lagrangian is that the $\theta_{a}$ field now appears as the $\tau$ component of a $U(1)$ gauge field coupling to a charge boson field $\phi$, with the dynamics of the $\theta_{a}$ field governed by $E_{\mathrm{cl}}$. In particular, in the presence of $B$ field, the nonmagnetic impurity appears as electric charge generating a static electric-field coupling to the charge bosons. Notice that the effective charge of the nonmagnetic impurity changes sign when it is moved from one sublattice to another, indicating that the "sign" of the charge is in fact a sublattice index. " $^{0,11}$

The logarithmic divergence in "electric-field" energy in the presence of nonmagnetic impurity in $E_{\mathrm{cl}}$ in two dimensions will be removed if bosons of opposite electric charge are nucleated from vacuum to screen the effective electric field, forming effective local magnetic moments around the impurity. The number of bosons nucleated from vacuum is $\sim 2 S$, as can be seen easily by counting the number of charges carried by the nonmagnetic impurity. The magnitude of magnetic moment formed around the impurity is thus $\sim S$. The energy cost for nucleating the bosons can be computed by solving the corresponding Schrödinger equation for charge bosons moving in scalar potential of external charge of magnitude $2 S e .{ }^{12}$ To capture qualitatively the physics, we estimate the energy cost by using a variational wave function $\psi(\vec{r}) \sim A e^{-|\vec{r}| / \xi_{1}}$, where $\xi_{1}$ is determined variationally. Minimizing the energy, we find that $\xi_{1}$ is of the order $\xi_{1} \sim\left[J S c^{2} /\left(g \mu_{B} B\right)^{3}\right]^{1 / 2}$. Notice that $\xi_{1} \rightarrow \infty$ as $B \rightarrow 0$ or $S \rightarrow \infty$, indicating that the formation of a magnetic moment around a nonmagnetic impurity is a nonperturbative quantum effect which cannot be captured by the usual spin-wave theory. The energy cost for nucleating the bosons is of the order $E_{1} \sim(2 S)\left[m+2 S B^{\prime 2} \ln \left(\xi_{1} / a_{o}\right)\right]$.

Next we discuss the situation when there is finite concentration of impurities $n$ randomly distributed in the system, which is the case of experimental interest. In our continuum theory, the presence of finite concentration of random impurities is equivalent to putting finite concentration of charges with magnitude $2 S B^{\prime}$ and random sign in our effective charge boson system. The behavior of the system is very different from the one impurity case, since now electric fields originating from opposite charges will cancel, with remaining electric-field energy of the order $\sim n E_{e}$, where $E_{e} \sim\left(2 S B^{\prime}\right)^{2} \ln \left(l / a_{o}\right)$ on average, $l \sim n^{-1 / d}$ is the average distance between nonmagnetic impurities. For $E_{e}>E_{1} \sim 2 S m$ or equivalently $l \gg \xi_{o} \sim a_{o} e^{J S /\left(2 g \mu_{B} B\right)}$, it is energetically favorable to nucleate charge bosons from the vacuum to screen the electric field, and magnetic moments will be formed around nonmagnetic impurities. The magnetic moments couple to each other weakly with $J_{\text {eff }} \sim J e^{-l / \xi_{1}}$ implying that Curie behavior will be present in magnetic susceptibility down to very low temperature $\sim J_{\text {eff }}$. As the concentration of nonmagnetic impurities increases, $l$ decreases until $l \sim \xi_{o}$. At this point a transition occurs where it becomes energetically unfavorable to nucleate bosons from the vacuum to screen the electric field, i.e., there will be no magnetic moments forming around nonmagnetic impurities at zero temperature when $l \leqslant \xi_{o}$. The boson-non-magnetic impurity bound states become excited states of the spin system.

The boson-non-magnetic impurity bound states can still be observed as effective local magnetic moments at finite temperature $T \geqslant m \sim g \mu_{B} B$ if $E_{e}+2 S m>E_{1}$ (corresponding roughly to $\left.\xi_{1}<l\right)$. At this energy range, the boson-impurity bound state appears as a stable excited state of the spin system with excitation energy $\sim\left(E_{1}-E_{e}\right) / 2 S<m$. At finite temperature $T \geqslant m$, these states will be occupied by thermally excited bosons, forming effective free magnetic moments around nonmagnetic impurities. It is important to emphasize that the formation of an excited boson bound state around a nonmagnetic impurity is only possible when there is a gap $m$ in the boson excitation spectrum. In the absence of nonmagnetic impurity, a direct computation of the spin-wave spectrum indicates that the spin-wave spectrum splits into two branches at small momentum $q$, with one branch carrying a gap larger than $m$ in our effective Lagrangian, and the other branch gapless as $q \rightarrow 0$. The contribution from the splitting of the two branches to $H_{1 / S}$ cancels to order $O\left(B^{2}\right)$, leading to our effective Lagrangian with only massive bosons. The splitting of the boson spectrum reappears if we include terms to order $O\left(B^{4}\right)$ in $H_{1 / S}$, and the boson bound state around the nonmagnetic impurity will cease to be a good eigenstate of the effective Lagrangian (the bound state turns into a resonant state). However, the decay rate of the resonant state $\sim B^{4}$ is small compared with the energy of the resonant state $\sim B^{2}$ in the limit of the weak magnetic field, indicating that the physical picture of the boson bound state around a nonmagnetic impurity is still a good approximation to the system in the weak-field limit.

As concentration of impurities increases and distances between impurities decreases further, the overlap between boson bound state wave functions at different impurity sites increases and a boson impurity band is formed (recall that the size of the boson bound state wave function is of the order $\left.\xi_{1}\right)$. For $l \leqslant \xi_{1}$, the bandwidth $W$ of this impurity band is of the order $\sim\left[\sqrt{m^{2}+(c / l)^{2}}-m\right]$ and the effective impurity potential strength is of the order $v \sim 2 S B^{\prime 2} /(1 / l)^{2}$. We find that the screening of effective impurity potential by charged bosons is weak and can be neglected. Estimating the scattering lifetime $\tau$ we find that bosons with energy $E \leqslant E_{l} \sim c l / \xi_{1}^{2}$ are strongly localized in this impurity band using the criteria $E \tau \leqslant 1$. Notice that the number of localized boson states $\sim n^{-1}$ and decreases as $n$ increases in this regime. The magnetic susceptibility will show Curie behavior at temperature above $T_{l} \sim E_{l} / k_{B}$. For $T \leqslant T_{l}$, the number of effective magnetic moments contributing to magnetic susceptibility decreases as temperature lowers. Roughly speaking, the contribution from nonmagnetic impurities to the magnetic susceptibility can be described by a magnetic-field and temperature-dependent density of local moments $n(B, T)$ which has the following properties: (i) $n(B, T) \sim 0$ at the region $g \mu_{B} B \gg k_{B} T$, (ii) $n(B, T) \sim n$ for $l \geqslant \xi_{1}$, and $\sim\left(n \xi_{1}^{4}\right)^{-1}$ for $l \leqslant \xi_{1}$ at the region $k_{B} T \gg g \mu_{B} B+E_{l}$, and is smoothly interpolating between the two regions. As temperature $T \rightarrow 0, n(B, T) \rightarrow 0$ and the contribution to magnetic susceptibility from impurities is of the order $\delta \chi \sim 4 S^{2} n \ln \left(l / a_{o}\right)$.

Our theory can be extended to three dimensions in a straightforward way. We find that in the weak-field limit, no magnetic moment is formed even in the limit of one single impurity at zero temperature because of the absence of infrared divergence associated with the Coulomb potential in 3D. However, bound states of bosons can be formed at 
$T \geqslant g \mu_{B} B$ as in the two-dimensional case, and can still result in Curie-type behavior in magnetic susceptibility for $l \gg \xi_{1} \sim\left(c / g \mu_{B} B\right)^{3}$. As concentration of impurities increases further, the boson bound states turn into impurity bands and the effective number of free magnetic moments $n(B, T)$ decreases when temperature is lower than the boson impurity band bandwidth as in two dimensions.

Summarizing, in this paper we have carried out an analysis of the effects of replacing spins by nonmagnetic impurities in Heisenberg antiferromagnets in a magnetic field in two and three dimensions in a semiclassical $1 / S$ expansion. We find within a continuum approximation that magnetic moments will form around nonmagnetic impurities once the magnetic field is put on the system, resulting in Curie-type behavior in magnetic susceptibility when concentration of impurities is not too high, and temperature is not too low.
Notice that our theory predicts that formation of local magnetic moments in the presence of external magnetic field and nonmagnetic impurities is a general behavior of ordered quantum antiferromagnets and is not restricted to particular materials. The theory explains the observation of Curie-type behavior in magnetic susceptibility in $\mathrm{La}_{2} \mathrm{Cu}_{1-x} \mathrm{Zn}_{x} \mathrm{O}_{4}$ and $\mathrm{Sr}\left(\mathrm{Cu}_{1-x} \mathrm{Zn}_{x}\right)_{2} \mathrm{O}_{3}$ compounds at temperatures well below Néel temperature. In particular, the vanishing of local moments at $T \leqslant g \mu_{B} B$ is a theoretical prediction which can be tested experimentally.

I thank G. Aeppli, M. Ma, and M. Azuma for very useful discussions and the hospitality of Lucent Technologies, Bell Labs. where part of this work was done. The work is partially supported by Hong Kong UGC Grant No. UST636/ 94P.
${ }^{1}$ Y. Fukuzumi et al., Phys. Rev. Lett. 76, 694 (1996).

${ }^{2}$ G. Xiao et al., Phys. Rev. B 35, 8782 (1987).

${ }^{3}$ H. Alloul et al., Phys. Rev. Lett. 67, 3140 (1991); K. Kakurai et al., Phys. Rev. B 48, 3485 (1993).

${ }^{4}$ S-W. Cheong et al., Phys. Rev. B 44, 9739 (1991); K. Uchinokura et al., Physica B 205, 234 (1995).

${ }^{5}$ N. Nagaosa and T.K. Ng, Phys. Rev. B 51, 15588 (1995).

${ }^{6}$ M. Azuma et al., Phys. Rev. Lett. 73, 3463 (1994).

${ }^{7}$ M. Azuma et al. (unpublished).

${ }^{8}$ N. Bulut et al., Phys. Rev. Lett. 62, 2192 (1989); see also N.
Nagaosa et al., J. Phys. Soc. Jpn. 58, 9781 (1989).

${ }^{9}$ It can be shown that the logarithmic divergence in classical linear response is removed once higher-order terms in $\theta_{s}$ and $\theta_{a}$ are included in the energy expression. The resulting classical energy is of order $B^{2} \ln \left(S J / \mu_{B} B\right)$.

${ }^{10}$ N. Read and S. Sachdev, Phys. Rev. B 42, 4568 (1990).

${ }^{11}$ T.K. Ng, Phys. Rev. B 52, 9491 (1995).

${ }^{12}$ The Schrödinger equation for charged boson in the effective scalar potential of a external point charge is $[E-V(r)]^{2} \psi(\vec{r})$ $=\left(c^{2} \nabla^{2}+m^{2}\right) \psi(\vec{r})$, where $V(r) \sim 2 S B^{\prime 2} \ln \left(r / a_{o}\right)$. 